Itinéraires Itinéraires

Littérature, textes, cultures

\title{
Modernités shakespeariennes : le cas de la traduction
}

\section{Marie Nadia Karsky}

\section{(2) OpenEdition}

1 Journals

\section{Édition électronique}

URL : http://journals.openedition.org/itineraires/1735

DOI : $10.4000 /$ itineraires. 1735

ISSN : 2427-920X

Éditeur

Pléiade

\section{Édition imprimée}

Date de publication : 1 décembre 2010

Pagination : 117-136

ISBN : 978-2-296-13183-5

ISSN : $2100-1340$

\section{Référence électronique}

Marie Nadia Karsky, « Modernités shakespeariennes : le cas de la traduction », Itinéraires [En ligne], 2010-4 | 2010, mis en ligne le 01 décembre 2010, consulté le 10 décembre 2020. URL : http:// journals.openedition.org/itineraires/1735; DOI : https://doi.org/10.4000/itineraires.1735

\section{(c) $(1) \odot \odot$}

Itinéraires est mis à disposition selon les termes de la licence Creative Commons Attribution - Pas d'Utilisation Commerciale - Pas de Modification 4.0 International. 


\title{
Modernités shakespeariennes : le cas de la traduction
}

\begin{abstract}
How is Shakespeare translated into French nowadays? And how do his translators contribute to making him modern? In this essay, we will examine and compare translations by Yves Bonnefoy, Jean-Michel Déprats, André Markowicz and Pascal Collin, raising the following questions: how is Shakespeare's verse rendered? To what effect do translators choose free or syllabic verse? How can translators do justice to the polysemy of Shakespeare's language? What becomes of notions which seem dated in $21^{\text {st }}$-century France? Through the use of a modern idiom, translators can adapt them to the present context, insuring that audiences understand what is at stake and laugh when the situation calls for it. However, modernizing the idiom does not necessarily involve conveying Shakespeare's poetry or the theatrical dimension of his plays. In French translations, making Shakespeare modern is thus less a matter of updating his idiom than of working on the poetic dimension of the French language, pushing back the limits of what is deemed receivable.
\end{abstract}

Keywords : rhythm, verse, gestus, polysemy, modernizing

Mots clés : rythme, vers, gestus, polysémie, modernisation

" Comme la mise en scène, la traduction de Shakespeare reste un champ d'expérimentation ouvert ${ }^{1}$. " Notre époque atteste, en France, d'un grand nombre de retraductions du théâtre et de la poésie de Shakespeare, tantôt faites dans le cadre ponctuel d'un spectacle, par le metteur en scène ou par un traducteur travaillant en étroite collaboration avec un directeur de troupe $^{2}$, tantôt provenant d'universitaires et de traducteurs. Là encore,

1. Jean-Michel Déprats, « Analyse comparative de plusieurs traductions françaises de Roméo et Juliette ", dans Nicole Vigouroux-Frey (dir.), Traduire le théâtre aujourd'hui ?, Rennes, Presses Universitaires de Rennes, coll. « Le Spectaculaire », 1993, p. 91-101, p. 100.

2. Ariane Mnouchkine a traduit trois pièces de Shakespeare pour les spectacles qu'elle a montés au début des années 1980 avec le Théâtre du Soleil. Pour ce qui est des collaborations, 
elles peuvent soit être occasionnelles, soit s'inscrire dans un projet de plus grande envergure, comme celui de la traduction des œuvres complètes ${ }^{3}$, et donner lieu ou non à des mises en scène. Quels sont les partis pris de ces nombreuses traductions et quelles sont les directions esthétiques qui se profilent?

Chaque époque relit un écrivain à l'aune des questions qu'elle se pose. Par les interrogations littéraires, dramaturgiques, métaphysiques, culturelles et sociales que ses œuvres font naître, Shakespeare se prête tout particulièrement à ces relectures. Comment les traductions récentes de ses œuvres contribuent-elles à inscrire le dramaturge dans la modernité? Fontelles systématiquement de Shakespeare " notre contemporain », et quelles sont les lectures de Shakespeare qui les sous-tendent?

La modernité se définit par ce qui, obéissant au changement constant, «fait de la crise une valeur, une morale contradictoire ${ }^{4}$ » et se place continuellement en rupture par rapport à ce qui précède. Les traductions obéissent à cette logique du changement : elles sont, pour la plupart, vite perçues comme caduques parce que la langue dans laquelle elles sont écrites n'éveille pas d'écho poétique à notre époque; leur renouvellement s'avère donc nécessaire. Henri Meschonnic signale toutefois que ce ne sont pas les traductions, mais notre pensée du langage et nos conceptions de la littérature qui vieillissent : lorsqu'elles sont fondées sur des notions de dualité et de discontinu (entre signifiant et signifié, oral et écrit), les traductions ne réussissent pas à faire entendre le rythme du texte, défini comme « l'organisation du mouvement de la parole dans le langage par un sujet » qui postule la continuité entre la lettre et le sens ${ }^{5}$. Les traductions des œuvres de Shakespeare peuvent-elles contribuer à repenser la théorie du langage et la conception de la littérature et s'inscrire, ainsi, dans une

on pense plus particulièrement à celle de Peter Brook et Jean-Claude Carrière ou, plus récemment, de Yann-Joël Collin et Pascal Collin. Outre Le Roi Lear (Montreuil, Éditions Théâtrales, 2007) et Le Songe d'une nuit d'été (Montreuil, Éditions Théâtrales, 2008), Pascal Collin a traduit les deux parties d'Henri IV, Richard III et les Sonnets (traductions non publiées en mars 2010).

3. Michel Grivelet et Gilles Monsarrat ont fait paraître les Euvres complètes de Shakespeare en 1995 chez R. Laffont (coll. « Bouquins ») dans une édition bilingue; la Bibliothèque de la Pléiade réédite depuis 2002 les Euvres complètes de Shakespeare, en édition bilingue également, sous la direction de Jean-Michel Déprats et de Gisèle Venet; les traductions de Shakespeare faites par André Markowicz paraissent aux éditions Les Solitaires Intempestifs et s'inscrivent elles aussi dans le projet d'une publication des œuvres complètes; quant aux nombreuses traductions d'Yves Bonnefoy, elles ont paru principalement chez Mercure de France et Gallimard.

4. Jean Baudrillard, entrée « Modernité » dans Encyclopaedia Universalis, 1995, p. 552-554, p. 552.

5. Henri Meschonnic, entrée « Rythme », dans Alain Rey (dir.), Dictionnaire culturel en langue française, Paris, Le Robert, 2005, p. 467. Voir aussi son Poétique du traduire, Paris, Verdier, 1999, p. 112-121. 
autre définition de la modernité que celle du changement constant? La traduction ne serait plus alors moderne du fait de l'écho de reconnaissance souvent passager qu'elle crée parmi le public récepteur, mais parce qu'elle deviendrait, par «sa prosodie, son rythme, sa signifiance ${ }^{6} »$, un texte littéraire à part entière.

Shakespeare pratiquait une relecture et une réécriture modernes des auteurs anciens, ouvrant, à partir d'éléments connus, sur des interrogations neuves. Comment les traducteurs actuels font-ils passer cette dimension de Shakespeare, poète et dramaturge moderne en ce qu'il repousse les frontières de ce qui, d'un point de vue linguistique, stylistique, culturel, était connu et acceptable à son époque?

Notre étude sera en partie axée sur la dimension théâtrale de la traduction et portera ainsi sur les traductions des pièces de Shakespeare et non de ses poèmes, bien que les Sonnets aient aussi été mis en scène ${ }^{7}$. Nous nous limiterons aux traductions faites en France, malgré l'intérêt que présentent, dans le cadre d'une étude sur la modernité, celles provenant d'espaces francophones, en particulier du Québec. Annie Brisset signale que les traductions, relativement peu nombreuses, de Shakespeare faites dans les années 1970 et 1980 au Québec sont liées à des considérations politiques : il importait de rendre audible une parole populaire et de créer une littérature québécoise plutôt que de faire entendre une littérature anglophone ou francophone venue d'ailleurs. Les pièces sont alors soumises à des processus de domestication où les références shakespeariennes sont soit omises, soit adaptées à l'histoire du Québec, afin de servir un discours identitaire ${ }^{8}$. Depuis, des traducteurs comme Normand Chaurette ou Antonine Maillet, restant plus fidèles à l'intrigue des pièces, soulignent les avantages qu'ils trouvent à se servir, pour leurs traductions de Shakespeare, du français québécois qui leur permet de faire entendre un idiome plus âpre et plus proche de la langue parlée ${ }^{9}$.

Un bref rappel historique de la traduction de Shakespeare en France nous permettra d'abord de resituer, contextuellement, les traductions récentes et les approches dont elles témoignent. Suite à cela, nous confronterons des traductions datant des cinquante dernières années en nous axant sur les trois grandes sources de difficultés présentées par Jean-

6. Henri Meschonnic, entrée « Traduction », dans Alain Rey (dir.), op. cit., p. 1504.

7. Entre autres par Peter Brook dans son spectacle intitulé « Love is my sin », qui s'appuyait sur une sélection des Sonnets dits par deux acteurs au Théâtre des Bouffes du Nord (Paris, avril 2009).

8. Annie Brisset, « L'identité en jeu ou le sujet social de la traduction », dans Nicole VigourouxFrey (dir.), op. cit., p. 11-21.

9. Chantal Gagnon, «Le Shakespeare québécois des années 1990 », Theatre Research in Canada / Recherches théâtrales au Canada, vol. 24, n 1-2, 2003, http://www.lib.unb.ca/ Texts/TRIC/TofC.html (consulté le 26 mars 2010). 
Michel Déprats ${ }^{10}$ : la question du vers à adopter, de la langue à choisir, modernisante ou non, enfin, de la nécessité de préserver, en traduction, la dimension théâtrale du texte. Nous nous appuierons sur le travail de quatre traducteurs aux approches distinctes : il s'agit d'Yves Bonnefoy, de JeanMichel Déprats, d'André Markowicz et de Pascal Collin. Les défis que pose la traduction de Shakespeare permettent à Yves Bonnefoy de creuser sa recherche poétique. André Markowicz s'attache à faire entendre le rythme du pentamètre iambique qu'il traduit par des décasyllabes. Jean-Michel Déprats part du principe qu'il faut faire passer, en traduction, la richesse de jeu caractéristique du texte shakespearien. La démarche de Pascal Collin consiste à rendre Shakespeare plus largement accessible à son public en se servant d'un idiome moderne et en soulignant les effets potentiellement comiques des textes ${ }^{11}$.

\section{De la prose au vers}

Shakespeare est introduit en France par Voltaire, dont la position à l'égard du dramaturge est ambivalente : admiratif du génie " plein de force et de fécondité, de naturel et de sublime »du poète ${ }^{12}$, il critique ce qu'il considère être son manque de goût et son ignorance des règles, estimant que si Shakespeare est tant apprécié en Angleterre, c'est que ses successeurs ont commis des fautes de goût encore pire que les siennes. Des adaptateurs chercheront, au cours des deux siècles qui suivent, à corriger ce qu'ils considèrent être, chez Shakespeare, d'abord un manque de goût, puis un défaut structurel. Dans le dernier tiers du XVIII ${ }^{\mathrm{e}}$ siècle, Jean-François Ducis écrit des adaptations en alexandrins des grandes tragédies où la fin est transformée afin d'assurer un dénouement heureux. En Angleterre également, les pièces de Shakespeare étaient souvent jouées dans des versions adaptées par des auteurs comme William Davenant ou John Dryden ${ }^{13}$. Malgré son admiration pour le théâtre de Shakespeare, Charles Lamb estimait qu'il ne convenait qu'à la lecture; le spectateur risquant d'être perturbé par le manque de réalisme des intrigues, il fallait

10. Jean-Michel Déprats, « Traduire Shakespeare », dans William Shakespeare, Tragédies, Paris, Gallimard, coll. « Bibliothèque de la Pléiade », 2002, p. 1xxxix-cxxi.

11. Yves Bonnefoy, Jean-Michel Déprats et André Markowicz ont traduit de nombreuses pièces de Shakespeare, qui ont souvent été mises en scène et se trouvent facilement en librairie dans leur version publiée. En revanche, à l'exception du Songe d'une nuit d'été qu'André Markowicz et Françoise Morvan ont traduit pour un spectacle, ces trois traducteurs ne traduisent habituellement pas Shakespeare en vue d'une mise en scène spécifique. L'inverse est vrai pour Pascal Collin, dont Le Roi Lear et Le Songe d'une nuit d'été ont été créées pour les mises en scène respectives de Jean-François Sivadier (Festival d'Avignon, 2007) et Yann-Joël Collin (Théâtre de l'Odéon, 2008).

12. Voltaire, Lettres philosophiques, dans Mélanges, Paris, Gallimard, coll. « Bibliothèque de la Pléiade », 1961, p. 81.

13. Gary Taylor, Reinventing Shakespeare [1989], Londres, Vintage, 1991, p. 29-30. 
les adapter pour la scène ${ }^{14}$. En France, Vigny a réécrit Othello en 1829 et Georges Sand remanié l'intrigue de As You Like It en 1856, afin de pallier ce qu'elle considère comme «l'absence à peu près totale de plan » de la pièce ${ }^{15}$. Shakespeare a ainsi connu en France un destin duel : pour la scène, ses pièces subissent une adaptation textuelle et structurelle; les traductions prévues pour la lecture, en revanche, respectent davantage l'intrigue. Après la parution de plusieurs pièces de Shakespeare traduites en prose par Antoine de La Place entre 1746 et 1749, Pierre Le Tourneur est le premier à traduire, en prose, les œuvres complètes de Shakespeare entre 1776 et 1783 . Dans sa préface, Le Tourneur déclare avoir cherché à garder les images et figures stylistiques des pièces, dont il traduit pourtant les vers par de la prose. Sa traduction obéit aux critères du « bon goût » de l'époque : les scènes sont ajustées aux règles du théâtre français classique et les images jugées choquantes sont modifiées pour passer sous silence toute référence sexuelle.

Au XIX ${ }^{e}$ siècle, les traductions de Le Tourneur sont révisées par François Guizot et Amédée Pichot dans les années 1820, puis elles sont suivies de celles, en prose toujours, de Benjamin Laroche (1839), d'Émile Montegut (1867), et de François-Victor Hugo (entre 1857 et 1872). La réception de Shakespeare prend un tournant en France avec cette traduction: le fils de Victor Hugo cherche à restituer, dans la prose dans laquelle il traduit, la richesse métaphorique de la langue de Shakespeare, sans modifier les images qui paraissent à l'époque choquantes ou incongrues. Jusqu'à la seconde moitié du $\mathrm{xx}^{\mathrm{e}}$ siècle, c'est en prose que Shakespeare est principalement traduit, malgré le rôle primordial du pentamètre iambique dans ses pièces. La première édition des Euvres complètes de Shakespeare dans la collection de la Pléiade témoigne encore de ce phénomène : la seule pièce dont les passages en vers sont rendus par des vers en français est Roméo et Juliette, traduit par Pierre Jean Jouve et Georges Pitoëff.

Ce n'est qu'au $\mathrm{xx}^{\mathrm{e}}$ siècle que les traducteurs auront progressivement recours au vers, principalement après la Seconde Guerre mondiale. Ainsi paraissent, en 1959, Jules César et Le Roi Lear, traduits en vers libres respectivement par Yves Bonnefoy et Armand Robin ${ }^{16}$. André Gide s'intéresse au contraste entre la concision propre à Shakespeare et l'allongement de la phrase qui résulte des traductions françaises, comparant le rythme du français à « [1'] appesantissement [d'un] tardigrade [qui] couvre en claudicant l'espace que le vers shakespearien a franchi d'un bond ${ }^{17} \gg$. S'il évoque ainsi le contraste rythmique entre le « resserrement » de l'original

14. D. F. Bratchell (ed.), Shakespearean Tragedy, Londres et New York, Routledge, 1990, p. 45.

15. Paul André Horguelin, Anthologie de la manière de traduire, Montréal, Linguatech, 1981, p. 168.

16. Pour l'édition bilingue des œuvres de Shakespeare au Club Français du Livre.

17. André Gide, "Avant-propos », dans William Shakespeare, CEuvres complètes, Paris, Gallimard, coll. « Bibliothèque de la Pléiade », 1959, p. ix-xv, p. ix. 
et la langue des traductions qui, par souci de logique et de clarté « devient explicative ${ }^{18}$ », il n'en explore jamais une des causes premières : le fait que les traducteurs ont rendu le vers par de la prose. En 2002, en revanche, l'option qui consisterait à traduire les vers par de la prose n'est même plus envisagée : Déprats consacre un long développement à la question de la traduction du vers shakespearien ${ }^{19}$. Depuis les traductions de Bonnefoy, la question qui se pose n'est donc plus celle du choix entre la prose et le vers, mais plutôt du vers à adopter afin de rendre la richesse stylistique et rythmique de l'original. Deux prises de position se dessinent : les partisans du vers libre s'opposent à ceux en faveur du vers syllabique. Yves Bonnefoy a principalement recours au vers libre, expliquant qu'il revient au poète et au traducteur de renouveler le vers lorsque les formes prédéfinies, comme l'alexandrin, n'assurent plus leur fonction poétique. Il met en garde contre les risques d'artificialité et de formalisme qui accompagnent le choix d'un vers régulier : privilégiant la monotonie rythmique, celui-ci détournerait l'attention du lecteur/spectateur de l'effet poétique du texte ${ }^{20}$. Étant donné que les systèmes métriques véhiculent des idéologies et des clichés esthétiques et culturels différents, il considère qu'il est impossible d'en remplacer un par un autre ${ }^{21}$. Si l'alexandrin se présente parfois malgré tout dans leurs traductions, Bonnefoy, et Déprats à sa suite, optent pour le vers libre dont la souplesse permet, mieux qu'un vers syllabique, de faire entendre la richesse poétique, dramatique, et rhétorique du vers de Shakespeare et la complexité de l'expérience humaine qu'il représente ${ }^{22}$. Bonnefoy a cependant souvent recours à l'hendécasyllabe, alexandrin tronqué d'une syllabe après l'hémistiche qui fait « nécessairement alterner le pair et l'impair, l'intemporel et le temps, l'Intelligible et l'incarnation ${ }^{23}$ " et réunit les contraires sans les amalgamer. On objectera, cependant, que le pentamètre iambique se caractérise par un schéma auditif tout à fait clair, tandis que le vers libre en est dénué. Le lecteur a tout le temps de voir, de relire, d'entendre et de peser le vers pour y discerner la plus grande récurrence d'un mètre, mais le spectateur perçoit-il à l'oreille le vers de onze syllabes, intermittent, de Bonnefoy? Ceci n'est peut-être pas le but de ce poète traducteur, pour lequel Shakespeare représente avant tout le terrain d'une recherche poétique ${ }^{24}$. Prenons deux exemples, du Roi Lear,

18. Ibid.

19. Jean-Michel Déprats, «Traduire Shakespeare », op. cit., p. xcic-ciii.

20. Yves Bonnefoy, La Communauté des Traducteurs, Strasbourg, Presses Universitaires de Strasbourg, 2000, p. 106. Voir aussi Jean-Michel Déprats, «Traduire Shakespeare », p. c-cii.

21. John Naughton (ed.), Shakespeare and the French poet: including an interview with Yves Bonnefoy, Chicago, Londres, The University of Chicago Press, 2004, p. 233-237.

22. Jean-Michel Déprats, «Traduire Shakespeare », op. cit., p. ci.

23. Yves Bonnefoy, op. cit., p. 109.

24. Ibid. p. 87. 
puis de Macbeth, illustrant la façon dont Bonnefoy se sert des différents vers pour créer des effets rythmiques et poétiques :

\begin{tabular}{|c|c|}
\hline Shakespeare & $\begin{array}{l}\text { My point and period will be throughly wrought, } \\
\text { Or well or ill, as this day's battle's fought }{ }^{25} \text {. }\end{array}$ \\
\hline \multirow[t]{3}{*}{ Bonnefoy } & Mon dessein, mon destin \\
\hline & Vont être en bien ou mal à jamais tranchés \\
\hline & Dans ce jour de combat - selon qui va gagn \\
\hline
\end{tabular}

Chez Shakespeare, la rime agit comme une conclusion, indiquant qu'une scène se termine ou permettant à l'acteur de marquer une légère pause après des paroles qui prennent ainsi une valeur de sentence. Les deux vers rimés de Bonnefoy, l'hendécasyllabe («Vont être en bien ou mal à jamais tranchés »), puis l'alexandrin qui clôt la scène, sont précédés d'un hémistiche isolé ("mon dessein, mon destin») sur lequel l'acteur peut ralentir, faisant jouer les assonances pour renforcer l'image d'une vie pesée dans la balance des dieux. Et dans l'extrait de Macbeth qui suit,

$\begin{array}{ll}\text { Shakespeare } & {[\ldots] \text { Whiles I threat, he lives: }} \\ \text { Words to the heat of deeds too cold breath gives. } \\ \text { (A bell rings) } \\ \text { I go, and it is done: the bell invites me. } \\ \text { Hear it not, Duncan; for it is a knell } \\ \text { That summons thee to Heaven, or to Hell }{ }^{26} \text {. } \\ \text { Mais je menace, je menace, et lui il vit. } \\ \text { Parler souffle sur l'acte et le refroidit. } \\ \text { La cloche sonne. } \\ \text { Les jeux sont faits, j'y vais. La cloche m'appelle. } \\ \text { Ne l'entends pas, Duncan, car c'est le glas, } \\ \text { Qui te réclame au Ciel, ou beaucoup plus bas. }\end{array}$

la répétition du syntagme verbal je menace permet à Bonnefoy de créer un alexandrin non régulier, immédiatement suivi de deux hendécasyllabes, puis d'un décasyllabe ( « Ne l'entends pas [...] glas »), la scène se terminant de nouveau sur un hendécasyllabe ( Qui te réclame [...] bas ») : les trois derniers vers, qui grâce aux nombreux monosyllabiques qui les composent, semblent plus rapides que l'alexandrin, contribuent au même titre que les jeux d'assonance et de rime à la légèreté et au rythme allant de ces vers.

25. William Shakespeare, King Lear, éd. R. A. Foakes, Londres et New York, Routledge, The Arden Shakespeare, 1997, IV.vii.96-97; William Shakespeare, Hamlet, Le Roi Lear, préface et trad. Yves Bonnefoy, Paris, Gallimard, coll. « Folio », 1978 [1965].

26. William Shakespeare, Macbeth, éd. Kenneth Muir, Londres, Thomson, The Arden Shakespeare, 2006 [1951], II.i.60-64; William Shakespeare, Macbeth, trad. Yves Bonnefoy, Paris, Mercure de France, 1983. 
S'appuyant sur les arguments de Bonnefoy, Jean-Michel Déprats a lui aussi recours au vers libre. L'absence de contrainte syllabique lui permet de rendre les nuances sémantiques et poétiques de l'anglais; en revanche, la légère pause à la fin de chaque vers libre fixe malgré tout une limite à sa longueur et lui impose une certaine rigueur. On trouve toutefois aussi des alexandrins dans ses traductions, grâce auxquels il rend, par exemple, l'effet de conclusion que produisent des vers rimés, comme dans le passage du Roi Lear déjà cité :

$\begin{array}{ll}\text { Shakespeare } & \text { My point and period will be throughly wrought, } \\ \text { Or well or ill, as this day's battle's fought. } & \\ \text { Déprats } & \text { Le combat d'aujourd'hui mettra un point final } \\ & \text { À ma vie, à son but, soit en bien soit en mal }{ }^{27} \text {. }\end{array}$

Les deux alexandrins rimés permettent à Déprats de créer un rythme insolite dans le contexte du vers libre dont il se sert par ailleurs. Il produit ainsi un effet de rupture grâce auquel l'acteur peut légèrement détacher ces vers du reste du texte. C'est également ce qui a lieu dans l'exemple de Macbeth déjà cité, où les alexandrins rimés donnent un ton plus solennel à la décision du personnage :

$\begin{array}{ll}\text { Shakespeare } & {[\ldots] \text { Whiles I threat, he lives: }} \\ \text { Words to the heat of deeds too cold breath gives. } \\ \text { (A bell rings) } \\ \text { I go, and it is done: the bell invites me. } \\ \text { Hear it not, Duncan; for it is a knell } \\ \text { That summons thee to Heaven, or to Hell. } \\ \text { [...] Mais, tandis que je menace, il vit. } \\ \text { Sous le souffle des mots, l'ardeur des actes refroidit. } \\ \text { Une cloche sonne. } \\ \text { J'y vais, et c'est fait. La cloche m'y convie. } \\ \text { N'écoute pas, Duncan, ce qui sonne dans l'air, } \\ \text { C'est un glas qui t'appelle au Ciel ou en enfer }{ }^{28} \text {. }\end{array}$

Partant du constat déjà énoncé par Gide selon lequel « Le français a besoin de plus de mots, de plus d'espace ${ }^{29}$ ", Déprats cherche à compenser en serrant de près la structure du texte shakespearien, en traduisant un vers par un autre vers, sans déborder sur celui qui suit lorsque Shakespeare ne le fait pas. Il en va autrement pour les partisans du vers syllabique, parmi lesquels on compte l'équipe des traducteurs réunie par Michel Grivelet et Gilles Monsarrat, ainsi qu'André Markowicz. Leur priorité

27. William Shakespeare, Le Roi Lear, dans Tragédies, trad. Jean-Michel Déprats, Paris, Gallimard, coll. «Bibliothèque de la Pléiade », 2002.

28. William Shakespeare, Macbeth, dans Tragédies, trad. Jean-Michel Déprats, Paris, Gallimard, coll. « Bibliothèque de la Pléiade », 2002.

29. Cité dans Jean-Michel Déprats, «Traduire Shakespeare », op. cit., p. lxxxiii. 
va à la transmission de l'impulsion rythmique du vers shakespearien. Dans la mesure où les spectateurs élisabéthains n'allaient pas voir, mais « entendre » une pièce (hear a play ${ }^{30}$ ), la dimension sonore du théâtre de Shakespeare est fondamentale. Ils optent cependant pour des vers différents : Grivelet et Monsarrat ont donné comme ligne directrice à leur édition la traduction du pentamètre iambique par un alexandrin libéré de sa forme classique, sans coupe à l'hémistiche ni alternance de rimes. Ils ont privilégié l'alexandrin pour sa portée traditionnellement poétique d'«homologue » du pentamètre iambique anglais dans la littérature française $^{31}$. André Markowicz a au contraire choisi le décasyllabe, qu'il considère par sa brièveté plus approprié à la restitution du rythme du vers shakespearien. Conscient des différences entre les systèmes métriques, syllabique du français, et accentuel et syllabique de l'anglais, Markowicz cherche à recréer le souffle du vers shakespearien et à reproduire une récurrence rythmique. La contrainte syllabique et le choix de la concision qu'implique le décasyllabe, poussent ce traducteur à se détacher parfois de la lettre du texte, à être moins exact par endroits que ne le sont des partisans du vers libre, d'étendre le sens sur deux vers ou au contraire, d'omettre des mots. Par exemple, les segments de vers de Lady Macbeth «Thou wouldst be great; / Art not without ambition », deviennent «Tu veux grandir; / Tu as de l'ambition ${ }^{32} »$ : Markowicz procède à une modulation par l'affirmative et par l'indicatif, ce qui lui permet certes de respecter la contrainte du décasyllabe, mais aux dépens de la cadence plus lente et pesée de l'original, où Lady Macbeth brosse un portrait peu flatteur, tout en hésitations et en velléités ${ }^{33}$, de son époux, la double négative not without participant de cette description d'un homme indécis.

Markowicz crée un vers énergique, à la fois rythmiquement régulier et poétiquement dense, qui s'appuie sur des effets d'allitérations et d'assonances parfois encore plus audibles dans la traduction que dans l'original, comme en témoignent les vers suivants du Songe d'une nuit d'été ${ }^{34}$ :

30. George Thaddeus Wright, Hearing the Measures : Shakespearean and Other Inflections, Madison, University of Wisconsin Press, 2001, p. xii.

31. Jean-Pierre Maquerlot, « Traduire Shakespeare aujourd'hui » (table ronde) dans Shakespeare et la France, études réunies par Patricia Dorval, Paris, Société Française Shakespeare, Actes du Congrès de 2000, p. 275-293, p. 278.

32. William Shakespeare, Macbeth, éd. Kenneth Muir, Londres, Thomson, The Arden Shakespeare, 2006 [1951], I.v.18-19; William Shakespeare, Macbeth, trad. André Markowicz, Besançon, Les Solitaires Intempestifs, 2008.

33. Le modal «wouldst » répété cinq fois en cinq vers (I.v.18-22), est ici traduit systématiquement par la forme indicative « tu veux » chez Markowicz.

34. William Shakespeare, A Midsummer Night's Dream, éd. Harold F. Brooks, Londres et New York, Routledge, The Arden Shakespeare, 1991 [1979], III.ii.19-24; William Shakespeare, Le Songe d'une nuit d'été, trad. André Markowicz et Françoise Morvan, Besançon, Les Solitaires Intempestifs, 2004. 
$\begin{array}{ll}\text { Shakespeare } & \text { And forth my mimic comes. When they him spy, } \\ \text { As wild geese that the creeping fowler eye, } \\ \text { Or russet-pated choughs, many in sort } \\ \text { (Rising, and cawing, at the gun's report), } \\ \text { Sever themselves, and madly sweep the sky, } \\ \text { So, at his sight, away his fellows fly } \\ \text { Mon pitre accourt; quand les autres le voient... } \\ \text { Mn coup de feu effraye moins les oies, } \\ \text { Gorge rouillée, les craves croasseurs } \\ \text { Froissent moins l'air quand ils voient le chasseur, } \\ \text { En noirs envols tout affolés d'effroi, } \\ \text { Que ses amis à lui quand ils le voient. }\end{array}$

La vivacité et la rapidité rythmique du décasyllabe de Markowicz, le travail de concision que ce vers implique, parfois aux dépens de la précision textuelle, offrent au lecteur français une traduction qui peut être ressentie comme plus immédiatement compréhensible que le texte de Shakespeare ne l'est pour des anglophones. Le recours à ce vers constitue ainsi une façon de "moderniser » Shakespeare, que cette modernisation relève, ou non, de la volonté consciente du traducteur.

\section{Polysémie et richesse poétique}

Confronter les textes introductifs de Gide et de Déprats nous permet de mieux cerner les changements qui se sont produits dans les traductions de Shakespeare au cours des cinquante dernières années. Nous avons vu que Gide ne posait pas la question de la traduction du vers, alors qu'elle occupe une place importante chez Jean-Michel Déprats. En revanche, Gide comme Déprats s'intéressent aux difficultés liées à la traduction de la polysémie lexicale, Gide parlant de «l'abondant foisonnement des suggestions » propre à la langue de Shakespeare ${ }^{35}$. Les sens multiples des mots sont activés en même temps en anglais, stimulant l'inventivité du traducteur. Prenons comme exemple de ce genre de polysémie l'expression « this child-changed father » (Le Roi Lear, IV.vii.17). Ces mots prononcés par Cordelia signifient à la fois « ce père transformé par ses enfants » et « ce père transformé en enfant ». Là où Pascal Collin ${ }^{36}$ et Jean-Michel Déprats privilégient chacun un des sens de l'expression, proposant respectivement " ce père changé en enfant » et « ce père tourmenté par ses enfants », Yves Bonnefoy traduit par le groupe ambivalent « ce père victime de l'enfant ». Le père est ici victime de son ou de ses enfants, mais l'usage de l'article défini suivi du singulier donne au syntagme l'enfant une extension plus large et plus générale, permettant de comprendre qu'il est aussi victime de l'enfant en lui.

35. André Gide, op. cit., p. xi.

36. William Shakespeare, Le Roi Lear, trad. Pascal Collin, Montreuil, Éditions Théâtrales, 2007. 
À ces difficultés liées à la polysémie lexicale se rajoute celle du décalage historique entre notre époque et celle de Shakespeare. Outre les mots vieillis ou dont le sens a changé, certains renvoient à une réalité qui nous est désormais inconnue ou qui n'est plus aussi évocatrice. Ainsi en est-il des adjectifs maidenly et maiden employés notamment dans Le Songe d'une nuit d'été. Dans les vers suivants, Helena reproche à Hermia de ne pas se comporter en jeune fille maidenly, c'est-à-dire chaste, virginale, pudique :

$\begin{array}{ll}\text { Shakespeare } & \text { It is not friendly, 'tis not maidenly (III.ii.217) } \\ & \text { Have you no modesty, no maiden shame (III.ii.285) } \\ \text { Déprats } & \text { Ce n'est pas amical, ce n'est pas virginal } \\ & \text { Êtes-vous sans vergogne, sans pudeur virginale }{ }^{37} \\ \text { Markowicz/Morvan } & \text { Ce n'est pas d'une vierge, d'une amie } \\ & \text { Ettes-vous donc sans pudeur virginale } \\ \text { Collin } & \text { Ce n'est pas amical, ce n'est pas digne d'une jeune femme } \\ & \text { N'as-tu aucune honte, aucune pudeur de jeune fille }\end{array}$

Déprats et Markowicz/Morvan gardent la référence à la notion de virginité, socialement et culturellement fondamentale à l'époque élisabéthaine, préservant la dimension historique du texte de Shakespeare que Pascal Collin, au contraire, gomme dans le choix qu'il fait d'actualiser la notion en «jeune fille» et même en «jeune femme». Abolissant la distance, l'actualisation offre au public un texte plus immédiatement compréhensible.

C'est également ce qui se produit pour la traduction d'une autre locution énigmatique, sweet marjoram, qu'Edgar donne en réponse à la demande de mot de passe que lui fait Lear au plus fort de sa folie (Le Roi Lear, IV.vi.93). Jean-Michel Déprats opte pour la traduction littérale par douce marjolaine, tandis qu'Yves Bonnefoy choisit gente marjolaine, connotant, par l'adjectif archaïque, un monde désuet, éloigné de nous, dont les codes nous paraissent obscurs. Pascal Collin traduit par tisane calmante, aux connotations ironiques immédiatement claires dans le contexte de cette scène extrêmement tendue. À l'époque élisabéthaine, la marjolaine servait à guérir les affections du cerveau, mais de nos jours, les propriétés apaisantes de cette plante ne sont pas connues de la moyenne des spectateurs anglophones, et le mot de passe prononcé par Edgar est énigmatique en anglais. Comme le faisait sans doute sweet marjoram du temps de Shakespeare, la locution tisane calmante dont se sert Collin laisse

37. William Shakespeare, Le Songe d'une nuit d'été, trad. Jean-Michel Déprats, Paris, Gallimard, coll. « Folio », 2003.

38. William Shakespeare, Le Songe d'une nuit d'été, trad. Pascal Collin, Montreuil, Éditions Théâtrales, 2008. 
supposer qu'Edgar émet un commentaire, à la fois attristé et ironique, sur la situation. Cette traduction ouvre donc sur une dimension tragicomique : une tisane serait-elle le minimum dont Lear aurait besoin à ce stade de sa folie, avant de sombrer dans le sommeil dont il se réveillera à la scène suivante? Le choix d'une traduction modernisante permet ici à Collin de faire entendre, sur un ton ironique et grinçant, l'humour qui point, chez Shakespeare, au plus fort des moments tragiques. Il peut ainsi souligner certaines des contradictions qui participent, aux yeux des lecteurs contemporains, de la modernité d'une pièce comme Le Roi Lear.

Les traducteurs actuels mettent souvent l'accent, bien plus que leurs prédécesseurs, sur les termes liés au corps et à la sexualité dans les pièces de Shakespeare ${ }^{39}$, créant parfois même des jeux de mot grivois pour compenser une perte. Ainsi en va-t-il de la traduction des paroles suivantes de Bottom, dans Le Songe d'une nuit d'été :

$\begin{array}{ll}\text { Shakespeare } & \begin{array}{l}\text { half his face must be seen through the lion's neck, } \\ \text { and he himself must speak through, (III.i.35-36) }\end{array} \\ \text { François-Victor Hugo } & \begin{array}{l}\text { qu'on voie la moitié de son visage à travers la } \\ \text { crinière du lion; il faudra que lui-même parle au } \\ \text { travers }\end{array} \\ \text { Déprats } & \begin{array}{l}\text { qu'on voie la moitié de son visage à travers le cou } \\ \text { du lion, et il faut que lui-même parle à travers }\end{array} \\ \text { Markowicz/Morvan } & \begin{array}{l}\text { la moitié de sa figure doit se voir derrière le cou du } \\ \text { lion, et, lui, il doit parler là du derrière }\end{array} \\ \text { Collin } & \begin{array}{l}\text { que la moitié de sa figure soit vue à travers le trou } \\ \text { du cou du lion, et il faut qu'il parle lui-même par le } \\ \text { trou du cou }\end{array}\end{array}$

Il y a bien ambiguité sémantique dans « and he himself must speak through [the neck] »: l'expression « to speak through the neck » signifiait, à l'époque élisabéthaine, parler à tort et à travers, faire des pataquès ${ }^{40}$, ce qui est justement le propre de Bottom. Ces mots n'évoquent en revanche rien de scatologique. Déprats, Markowicz/Morvan et Collin traduisent tous de façon littérale à l'aide du mot cou, permettant à l'acteur de jouer sur les sonorités du mot, ce que ne s'autorisait pas François-Victor Hugo, par exemple (dont nous avons cité ici la traduction afin de mieux souligner la différence entre les versions). Déprats reste discret, le choix de jouer sur les sonorités relève de l'acteur; en revanche, Markowicz/Morvan et surtout Collin poussent plus loin la plaisanterie verbale qui, chez eux, s'entend inévitablement. En jouant sur deux des trois natures grammaticales du mot derrière, d'abord préposition puis substantif, pour traduire la préposition

39. Jean-Michel Déprats, «Traduire Shakespeare», op. cit., p. xcvii.

40. D'après l'entrée au mot « neck » dans le dictionnaire Oxford English Dictionary, 1989. 
through, Markowicz/Morvan présentent un personnage amusant par sa naïveté; dans la traduction de Collin, en revanche, la même expression aux consonances potentiellement grossières est répétée, créant un effet légèrement monotone absent du texte de Shakespeare, et qui contribue à rendre Bottom plus clairement trivial que chez les autres traducteurs.

Pascal Collin a recours à un idiome moderne qui se caractérise par des effets de contraste entre un lexique parfois recherché et un vocabulaire qui relève d'un registre soudain familier, voire, parfois, grossier. Ainsi, dans Le Roi Lear, Collin ne traduit pas boarish dans " boarish fangs " (III.vii.57) par sanglier (choix fait par Déprats et Bonnefoy) mais par le terme plus inattendu de phacochère : cousin africain du sanglier, le phacochère ne prendra son nom en français et en anglais (warthog) qu'au $\mathrm{XIX}^{\mathrm{e}}$ siècle. Sans doute Collin a-t-il préféré phacochère à sanglier pour des raisons de sonorités, le mot pouvant être projeté par l'acteur de façon retentissante. De même, la question du duc d'Albany, « where was his son when they did take his eyes? »(Le Roi Lear, IV.ii.89), tout en simplicité par ses monosyllabes et ses mots d'origine anglo-saxonne, prend une tonalité presque pédante lorsque Collin se sert du verbe énucléer : «Où était son fils pendant qu'on l'énucléait ${ }^{41}$ ? » À l'opposé, il n'hésite pas à se servir de mots qui rendent le registre plus familier que ce n'est le cas chez Shakespeare. En témoigne sa traduction du cri de Lear dans la tempête :

$\begin{array}{ll}\text { Shakespeare } & \text { Strike flat the thick rotundity o' the world (III.ii.7) } \\ \text { Bonnefoy } & \text { Aplatis de ton choc l'énorme sphère du monde } \\ \text { Déprats } & \text { Aplatis l'épaisse rotondité du monde } \\ \text { Collin } & \text { aplatis comme une crêpe la sphère épaisse de la terre }\end{array}$

Par l'ajout de la comparaison « comme une crêpe », Collin privilégie, à un moment de grande charge poétique, une expression stéréotypée relevant du registre courant. On peut s'interroger sur le but d'un tel décalage : s'agit-il pour Collin de renforcer, par l'élément de comparaison, l'effet imagé de la locution? Ou plutôt, ce qui semble plus probable, d'ajouter aux imprécations de Lear une nuance comique qui participerait d'une certaine modernité au théâtre en créant un effet de distanciation pour le spectateur, malgré l'absence d'un effet de ce genre à cet endroit précis du texte anglais?

C'est dans Le Songe d'une nuit d'été que le nivellement par le bas du registre de Collin est le plus audible. La forêt d'Athènes, avec ses amants qui se disputent ainsi que ses fées et ses elfes, semble chez lui peuplée de jeunes citadins du début des années 2000. Des expressions modernes

41. Bonnefoy et Déprats proposent tous deux : «Où était son fils quand on lui a arraché les yeux?» 
et familières comme «tu t'es complètement planté » pour « Thou hast mistaken quite » (III.ii.88) lancé par Oberon, roi des fées, à son serviteur Puck, « lâche moi la grappe » pour « let loose » (III.ii.260), « Tu te donnes trop de mal / pour une qui se fout bien de toi et de tes services » pour « You are too officious / In her behalf that scorns your services » (III. ii.330-331), «tout ce foutoir est de ta faute » pour « all this coil is long of you » (III.ii.339) nous permettent certes d'entendre l'émotion ou la colère des différents personnages, mais elles neutralisent leurs différences en leur donnant, à tous, le même langage. Or Shakespeare a construit la scène de dispute dans la forêt sur un décalage stylistique entre une situation de confusion absurde et son expression poétique dont l'artificialité est rehaussée par un vers régulier et souvent rimé. Si en anglais, la scène tout entière avec ses quiproquos et sa volée d'insultes provoque les rires des spectateurs, cela provient en grande partie des invectives hautes en couleurs, qui ne sont pourtant pas grossières. De plus, les personnages ne s'expriment pas de la même manière que les spectateurs : la distance entre les personnages et le public est justement renforcée par l'aspect artificiel de la scène, lié à son expression poétique fortement marquée. Le registre populaire fréquemment entendu chez Collin, dans son Roi Lear comme dans son Songe d'une nuit d'été, participe du comique dans le contexte d'une mise en scène particulière. Shakespeare en paraît peut-être plus moderne car plus proche de la langue courante, voire familière, pratiquée et/ou comprise à notre époque, mais cela se fait parfois au détriment de la subtilité lexicale et poétique de l'original.

\section{Un texte pour la scène}

Depuis une trentaine d'années se profile plus nettement la question du fonctionnement scénique des traductions de Shakespeare. Gide observait que le spectateur n'avait pas le loisir de s'interroger comme le faisait le lecteur sur les multiples sens d'un texte dit par des acteurs, suggérant que Shakespeare comptait sur l'élan produit par le spectacle, le « mouvement précipité de l'action » pour faire passer « ce qui [...] gênerait à la lecture ${ }^{42}$ ». La question de l'efficacité dramatique de Shakespeare en français s'était posée dès les premières traductions de ses œuvres au XVIII ${ }^{\mathrm{e}}$ siècle, une des réponses étant, on l'a vu, l'adaptation de ses pièces pour la scène. Comment, cependant, traduire - et non adapter - Shakespeare de façon à assurer son efficacité théâtrale? Cette question se pose aux traducteurs actuels, en général très soucieux de rendre la dimension théâtrale de Shakespeare. Non qu'ils traduisent nécessairement en vue d'une mise en scène spécifique ${ }^{43}$,

42. André Gide, op. cit., p. xiv.

43. Le cas se présente toutefois aussi : Pascal Collin traduit pour des mises en scène, c'est également ce qui s'est produit pour André Markowicz et Françoise Morvan avec Le Songe d'une nuit d'été. 
mais leurs traductions se font en fonction de la «situation d'énonciation virtuelle $[\ldots]$ du futur public [...]. Le traducteur est dans la position d'un lecteur et d'un dramaturge (au sens technique du mot) : il fait son choix dans les virtualités et les parcours possibles du texte à traduire ${ }^{44} \gg$. Le concept brechtien de gestus est utile pour comprendre certains choix de traduction au cours des dernières années. Bertolt Brecht définit le gestus de deux façons. D'abord, comme «un ensemble de gestes, de jeux de physionomie et (le plus souvent) de déclarations faites par une ou plusieurs personnes à l'adresse d'une ou de plusieurs autres ${ }^{45} »$. Le gestus est donc social, témoignant des rapports entre les individus et situant l'homme dans sa relation aux autres. S'il peut ouvrir sur une gestuelle, il ne se confond ni ne se limite aux gestes et aux paroles : Brecht explique que « les mots et les gestes peuvent être remplacés sans que le gestus change pour autant ${ }^{46} »$. Le gestus brechtien, attitude physique et mentale qui suppose des rapports sociaux, ouvre sur des possibilités de jeu et de mise en scène qui, réalisées ou non, trouvent leur source dans le texte; il revient donc au traducteur de les reproduire.

Le deuxième sens du gestus brechtien est repris par Jean-Michel Déprats lorsqu'il en évoque l'importance pour la traduction théâtrale, citant Brecht :

La phrase «Arrache l'œil qui t'est un objet de scandale » est d'un point de vue gestuel moins riche [explique Brecht] que la phrase : «Si ton œil t'est un objet de scandale, arrache-le». Dans celle-ci, on montre d'abord l'œil, puis vient la première partie de la phrase, qui contient manifestement le gestus d'une hypothèse; enfin arrive la seconde partie, comme une attaque-surprise, un conseil libérateur ${ }^{47}$.

Le gestus se conçoit ici comme des possibilités de jeu corporel et vocal offertes à l'acteur par le rythme, la syntaxe, la prosodie et les choix lexicaux du texte, possibilités que le traducteur doit s'attacher à rendre dans la langue cible. Le sens social n'est plus celui qui prime; le gestus tel qu'il est repris par Déprats se définit plutôt comme les éléments textuels dans lesquels acteur et metteur en scène trouvent un matériau de jeu. Patrice Pavis rappelle que «mot et geste forment au théâtre une unité dialectique qu'on ne saurait disjoindre ${ }^{48} »$; il invente le terme de verbo-corps pour évoquer l'«alliance du texte prononcé et des gestes (vocaux et physiques) accompagnant son énonciation, [le] lien spécifique que le texte entretient

44. Patrice Pavis, Dictionnaire du Théâtre, édition revue et corrigée, Paris, Dunod, 1996, p. 386 .

45. Bertolt Brecht, L'Art du comédien. Écrits sur le théâtre, Paris, L'Arche, 1999, p. 124.

46. Ibid.

47. Jean-Michel Déprats, «Traduire Shakespeare », op. cit., p. cvii.

48. Patrice Pavis, Le Théâtre au croisement des cultures, Paris, José Corti, 1990, p. 155. 
avec le geste ${ }^{49}$ ». Pavis s'intéresse à la façon dont le lecteur, l'acteur, et donc aussi le traducteur, s'imaginent, à partir de la lettre du texte, le déroulement d'une scène, ce qu'il appelle la mise en jeu. Tant pour Pavis que pour Déprats, il est indispensable que le traducteur imagine cette mise en jeu comme préalable non seulement à la mise en scène, mais surtout à la traduction.

Quelques exemples permettront d'illustrer l'usage que les traducteurs font du concept du gestus, d'abord en tant qu'attitude envers autrui, puis envisagé comme potentiel de jeu que le texte offre aux acteurs.

L'usage que les personnages shakespeariens font des pronoms, passant de thou à you ou inversement pour s'adresser à la même personne, peut déconcerter le spectateur et le lecteur actuels. Pourtant, parce qu'il implique une modification dans la façon d'envisager autrui, il témoigne d'un changement de gestus social, d'un point de vue nouveau porté sur une situation. Ainsi, Le Roi Lear offre un exemple d'alternance intéressant par sa richesse gestique : la familiarité de la question « how dost, my lord? » que Regan pose à Gloucester s'oppose à l'usage de la forme polie du possessif de deuxième personne du pluriel your dont elle se sert juste après. Ce passage d'une forme à l'autre peut refléter une désinvolture et une agressivité latentes qui offrent un potentiel de jeu intéressant pour les acteurs.

$\begin{array}{ll}\text { Shakespeare } & \text { How dost, my lord? } \\ & {[\ldots] \text { What, did my father's godson seek your life? (II.i.89 }} \\ \text { Bonnefoy } & \text { Comment allez-vous, monseigneur? } \\ & {[\ldots] \text { Est-ce donc vrai? Le filleul de mon père }} \\ & \text { En voulait à vos jours? } \\ \text { Déprats } & \text { Comment va, mon seigneur? } \\ & {[\ldots] \text { Quoi, le filleul de mon père en veut à votre vie? }} \\ \text { Collin } & \text { Comment va monseigneur? } \\ & {[\ldots] \text { Quoi, le filleul de mon père, attenter à votre vie? }}\end{array}$

Regan use d'un tutoiement qu'elle ne reprendra que plus tard, lors de l'interrogatoire et de la torture auxquels elle soumettra le comte. L'hypocrisie de Regan et de son mari, leur attitude faussement respectueuse et cordiale (audibles dans les locutions my noble friend (mon noble ami), good old friend (bon, vieil ami) que Shakespeare leur attribue de façon répétée) sont d'emblée soulignées par cette marque de familiarité surprenante et inconvenante ici, mais tout à fait en accord avec la locution the old man (le vieillard) dont Regan se servira pour parler de Gloucester, en sa présence, à la fin de la même scène. En traduisant par une question parfaitement polie qui maintient le vouvoiement, Bonnefoy ne rend pas ce décalage. Chez Collin, Regan s'adresse à Gloucester à la troisième personne, marque 
de politesse et de sollicitude accrue. En revanche, la virgule ajoutée par Déprats ( « comment va, mon seigneur ») rend bien la familiarité grossière de la réplique, soulignant la rupture de gestus social à laquelle invitent ces mots de Regan. Ainsi, le respect de la lettre du texte, visible ici dans le changement de pronom, permet à la traduction de Déprats de refléter ces changements subits, et subtils, de gestus (au sens d'attitude envers autrui) et de proposer un texte riche d'un point de vue théâtral, car ambigu.

Le rythme du vers participe lui aussi du gestus (dans le sens, cette fois, de verbo-corps), ce que rappellent des metteurs en scène comme John Barton, Peter Brook, Peter Hall et Maik Hamburger, qui soulignent tous les écueils d'une diction naturalisante où le sens prime sur la forme, et donc sur le vers : " les comédiens se mirent à confondre vers et langage quotidien, croyant qu'il suffisait de parler "comme dans la vie". Ainsi les vers devenaient ordinaires et fades en raison d'une diction quelconque, indifférente ${ }^{50}$.» Partant du point de vue selon lequel les textes de théâtre sont des masses de sonorités et de formes rhétoriques, Peter Hall indique que faire résonner le vers, en souligner le rythme par la mise en relief de certains éléments ouvre, pour l'acteur et les spectateurs, sur des sens nouveaux ${ }^{51}$. Les irrégularités métriques donnent vie au vers shakespearien, et il importe que les traducteurs créent un rythme à la régularité irrégulière, elle aussi ${ }^{52}$.

Ainsi, dans l'exemple suivant extrait du Songe d'une nuit d'été, les injures proférées par Lysander à Hermia consistent en monosyllabiques : portant chacun un accent de mot, ils exigent une déclamation plus lente ${ }^{53}$. L'acteur est donc amené à ralentir sur le premier vers, d'autant plus qu'on y entend au moins un spondée dans « vile thing ${ }^{54}$ »:

Shakespeare
$\begin{aligned} & \text { Hang off, thou cat, thou bur; vile thing, let loose, } \\ & \text { Or I will shake thee from me like a serpent. (III.ii.260-261) }\end{aligned}$
$\begin{aligned} & \text { Léprats } \\ & \text { Ou je vais te rejeter loin de moi comme un serpent. }\end{aligned}$
Markowicz/Morvan
$\begin{aligned} & \text { Bas les pattes, chat noir, teigne puante, } \\ & \text { Ou je te jette au loin, comme un serpent. }\end{aligned}$

50. Peter Brook, op. cit., p. 15. Voir aussi John Barton, Playing Shakespeare, Londres, Methuen, 1984; Peter Hall, Shakespeare's Advice to the Players, Londres, Oberon Books, 2003; Maik Hamburger, «If it be now : the Knocking of Fate - Reading Shakespeare for Translation », dans Rui Carvalho Honem et Ron Hoenselaars, Translating Shakespeare for the 21st Century, Amsterdam et New York, Rodopi, 2004, p. 117-128.

51. Peter Hall, op. cit., p. 24.

52. Maik Hamburger, op. cit., p. 128.

53. John Barton, op. cit., p. 197.

54. Selon la décision de l'acteur, la voyelle diphtonguée de thou peut porter un accent tonique ou non. On peut dire thoù càt (en accentuant les deux mots) ou thou càt (en n'accentuant que le substantif). Quoi qu'il en soit, la diphtongue implique un ralentissement du débit. 
Collin Bas les pattes, espèce de chatte, sale teigne!

Lâche-moi la grappe, chose répugnante,

ou je vais te secouer pour t'arracher à moi, comme un serpent.

En se servant des locutions espèce de et sale, Collin traduit idiomatiquement le pronom thou qui précède les noms. Cette exactitude rallonge cependant son texte d'un vers par rapport à l'original. Les quatre termes plurisyllabiques dont il se sert (espèce de, répugnante, secouer, t'arracher) contribuent à étirer sa traduction par rapport au texte de Shakespeare. Le choix du décasyllabe contraint en revanche Markowicz/Morvan à se défaire de la moitié d'un vers («vile thing, let loose ») tout en rajoutant des adjectifs (noir, puante) pour compenser l'impossibilité de traduire le pronom. Déprats privilégie, lui aussi, des monosyllabiques; il joue sur les assonances en [a] (chatte, lâche moi) et crée un ralentissement rythmique sur les deux substantifs chatte, ronce qui peut constituer un équivalent du spondée.

Les traductions du demi-vers «out, out; brief candle» de Macbeth (V.v.24) se prêtent également à une analyse intéressante du point de vue rythmique. La préposition adverbiale out prend la fonction d'un impératif, effet impossible à reproduire littéralement en français. Déprats reste fidèle à son exigence de précision par rapport au nombre de mots et traduit en doublant l'impératif : " éteins-toi, éteins-toi, courte flamme ». Bonnefoy, en revanche, privilégie le rythme créé par les deux premières syllabes chez Shakespeare: "éteins-toi, brève lampe!» Markowicz calque un des sens de la préposition dans " dehors, / dehors, brève bougie ». Son choix provient-il du désir de faire entendre un reliquat de l'anglais dans sa traduction? D'introduire « l'étranger ${ }^{55}$ » dans la langue d'arrivée? Quelle qu'en soit la raison, out a dans ce vers le sens d'éteindre, non de faire sortir, et sa traduction par dehors donne au mot un sens et un poids qu'il n'a pas dans l'original. Des phénomènes de ce genre se produisent parfois dans les traductions de Markowicz, comme s'il cherchait soudain à créer un effet de trouble dans un texte par ailleurs limpide.

Les traducteurs contemporains s'attachent à rendre la dimension auditive et articulatoire des textes shakespeariens et les effets stylistiques et dramatiques ainsi créés. Jean-Michel Déprats met l'accent sur l'importance de la dimension sensible et sensorielle des mots chez Shakespeare, sur le problème de leur plus grande brièveté par rapport au français, et surtout de la richesse connotative qui provient de leurs origines différentes (latine ou anglo-saxonne), dimension dont le français est dépourvu. Pour pallier ce manque, Jean-Michel Déprats respecte, dans la mesure du possible, l'ordre des syntagmes de l'anglais, par exemple dans le vers : « you speak not as

55. Au sens où l'entend Antoine Berman dans L'Épreuve de l'étranger : culture et traduction dans l'Allemagne romantique, Paris, Gallimard, 1984 et dans La traduction et la lettre ou l'auberge du lointain, Paris, Seuil, 1999. 
you think » (Le Songe d'une nuit d'été, III.ii.191) : « vous ne dites pas ce que vous pensez », à l'inverse de Collin, «Tu ne penses pas ce que tu dis », et de Markowicz/Morvan, « Non, vous ne pouvez pas penser ces mots». Ce respect du texte anglais a aussi des conséquences sur le jeu des acteurs, comme dans ce vers de Macbeth,

$\begin{array}{ll}\text { Shakespeare } & \text { The Queen, my lord, is dead. (V.v.16) } \\ \text { Bonnefoy } & \text { La Reine, monseigneur! Elle est morte. } \\ \text { Déprats } & \text { La reine, mon seigneur, est morte. } \\ \text { Markowicz } & \text { La reine, monseigneur, est morte. }\end{array}$

où, par leur respect de l'ordre des mots et de la ponctuation, Déprats et Markowicz offrent à l'acteur la possibilité de dire cette phrase de plusieurs tons différents (annonce laconique, factuelle, ton plus inquiet...). Les modifications apportées par Bonnefoy (le point d'exclamation et la reprise du sujet par le pronom elle) rendent au contraire le laconisme impossible. Bonnefoy interprète le vers davantage, lui donnant une direction de jeu pour l'acteur et permettant au lecteur d'entendre un sentiment d'effroi chez le serviteur, alors que Déprats et Markowicz laissent le champ libre à l'acteur et au metteur en scène.

Nous avons tenté d'apporter des éléments de réponse à la question de savoir comment les traducteurs inscrivent Shakespeare dans la modernité. Ces cinquante dernières années, on a pu observer deux moments de rupture par rapport à l'histoire de ses traductions en France : le vers de Shakespeare n'est plus traduit par de la prose mais par un vers, ce qui pose la question du choix entre le vers régulier et le vers libre; Shakespeare est certes perçu comme poète, mais comme dramaturge avant tout, écrivant pour le corps et la voix des acteurs. Les exemples cités ici auront permis de présenter quelques unes des formes que prend le travail sur la langue effectué par les traducteurs. Par ses choix lexicaux et syntaxiques, Pascal Collin ancre le texte de Shakespeare dans l'époque actuelle, il fait ressortir l'aspect comique ou ironique des pièces qu'il traduit en privilégiant par endroits les termes prosaïques et en recherchant la proximité sonore entre l'anglais et le français. Collin rend ainsi Shakespeare plus contemporain de son public et ainsi plus accessible. Les traductions qui s'appuient sur une langue actuelle et courante sont certes vite datées, ce dont Collin est pleinement conscient ${ }^{56}$, mais la dimension transitoire de la traduction participe peutêtre, justement, de sa modernité, dans la mesure où celle-ci est synonyme de changement, d'éphémère ${ }^{57}$.

56. Pascal Collin, «Traduire pour le plateau », dans William Shakespeare, Le Roi Lear, Montreuil, Éditions Théâtrales, 2007, p. 12-15, p. 12.

57. Jean Baudrillard, op. cit., p. 552. 
Or inscrire Shakespeare dans la modernité ne signifie pas nécessairement en moderniser le lexique et la syntaxe. L'idiome choisi par les autres traducteurs n'est pas toujours aussi délibérément contemporain que celui de Collin. La mise en relation de l'avant-propos de Gide et du Traduire Shakespeare de Déprats, qui a servi de toile de fond à cette étude, révèle combien notre rapport à l'écriture et à la traduction a changé. Gide partait du principe que les différences stylistiques, linguistiques, et culturelles entre le français et l'anglais ne permettaient pas de traduire Shakespeare dans un français élégant sans le trahir ${ }^{58}$. S'ils sont d'accord avec ce principe, les traducteurs actuels s'inscrivent dans la lignée des travaux d'Antoine Berman et d'Henri Meschonnic, cherchant à travailler l'expressivité poétique de la langue cible et à rendre audibles l'étrangeté de la langue et de la poésie de Shakespeare, son rythme et sa densité. Ils évitent de lisser la traduction mais cherchent au contraire parfois même à la faire ressortir en tant que telle.

Une traduction semble en appeler une autre : insatisfaits face à ce qui n'a pas pu être rendu, les traducteurs sont poussés à retraduire Shakespeare. Les approches différentes contribuent à notre compréhension des pièces : Collin les rend plus actuelles, Bonnefoy et Markowicz permettent de réfléchir à la tension entre deux langages poétiques très différents, et Déprats rend manifeste la richesse lexicale, stylistique, rhétorique et scénique des textes shakespeariens. Par son écoute minutieuse du texte et son effort pour serrer la langue de Shakespeare au plus près, il parvient dans ses traductions à ce qu'Yves Bonnefoy appelait de ses vœux : le « forcement que le traducteur se doit d'opérer sur la langue de son époque [... $]^{59} \gg$. Malgré les zones d'ombre qui subsistent, la lecture de Shakespeare dans l'original s'enrichit, elle aussi, de ces diverses traductions : le lecteur peut revenir sur des passages dont la compréhension, devenue parfois incertaine ou trop automatique en anglais, s'éclaire et s'affine grâce à la confrontation des traductions entre elles.

\section{Marie Nadia Karsky \\ Université de Paris 8 Vincennes - Saint-Denis}

58. André Gide, op. cit., p. ix et xv.

59. Yves Bonnefoy, La Communauté des traducteurs, op. cit., p. 92. 